\title{
Prevalence and antibiotic resistance of uropathogens in children with urinary tract infections referring to Abuzar hospital in Ahvaz
}

\author{
Sousan Akrami1,2, Seyede Mahla Mavalizade2,3, Mehran Varnaseri Ghandali4, Aram Asareh Zadegan \\ Dezfuli $^{1}$, Zahra Farshadzadeh $^{1}$, Arshid Yousefi Avarvand $3{ }^{, *}$ \\ 1Department of Microbiology, School of Medicine, Ahvaz Jundishapur University of Medical Sciences, Ahvaz, Iran \\ 2Student Research Committee, Ahvaz Jundishapur University of Medical Sciences, Ahvaz, Iran \\ 3Department of Laboratory Sciences, School of Allied Medical Sciences, Ahvaz Jundishapur University of Medical Sciences, Ahvaz, Iran \\ 4Department of Infectious Diseases, Razi Hospital, Ahvaz Jundishapur University of Medical Sciences, Ahvaz, Iran
}

\begin{abstract}
Urinary tract infection (UTI) is a prevalent disease among children. This study is an attempt to find the bacterial agents of UTI and antibiotic resistance in children. A descriptive cross-sectional study was carried out on 1316 clinical samples of children at Abuzar Hospital in Ahvaz that had positive urine culture. The strains were determined through biochemical tests and differential culture media. The pattern of antibiotic resistance of the studied strains was determined by the disk diffusion method. Out of the 1316 children surveyed, 821 were girls and 495 were boys. The most isolated strains from urine cultures were Escherichia coli $(57.52 \%)$ followed by Enterococcus (12.15\%). E. coli isolates demonstrated the highest resistance to ampicillin (57.06\%) and amikacin was recognized as the most effective antibiotic with a sensitivity of $91.94 \%$. E. coli was the most common causative agent of UTI in children. Amikacin was recognized as the appropriate choice against urinary tract pathogens. Because, in different regions and over time, the frequency distribution of antibiotic resistance varies, it is recommended to carry out periodic monitoring of antibiotic resistance for infection control.
\end{abstract}

Keywords: Urinary tract infection, Disc diffusion, Antibiotic resistance

\section{Introduction}

Urinary tract infections (UTIs), a common microbial infection, occurs at most ages, especially in children and infants [1]. These infections can be in the lower and upper urinary tract or both [2]. In general, UTI in females are more prevalent (3-5\%) compared to males (1\%); however, boys at one year of age are at higher risk of developing the infections [3, 4]. The UTI is a major cause of fever and referral to the emergency wards and hospitalization, especially in infants [5]. This disease can cause serious complications such as urinary disorders, uremia, high blood pressure, and even death [6]. About 150 million UTI occur worldwide each year. These infections are among the main reasons for admission to hospitals and also imposing medical costs in these centers [7].

The most common etiological cause of UTI is Escherichia coli [8], which can be due to the greater ability of this bacterium to bind to urinary tract cells, greater resistance to antibacterial properties of human serum, production of hemolysin, and increased production of capsule antigen [9]. Due to the high prevalence and serious complications, diagnosis and treatment of UTI in children are necessary as soon as

\footnotetext{
* Arshid Yousefi Avarvand, Ph.D

Department of Medical Laboratory Sciences, School of Para Medicine, Ahvaz Jundishapur University of Medical Sciences, Ahvaz, Iran

Tel/Fax: +98 6133738317

Email: arshid.yousefi5@gmail.com

https://orcid.org/oooo-0oo2-3987-9820
}

Received: May, 04, 2021

Accepted: June, 26, 2021
(C) The Author(s) 2021

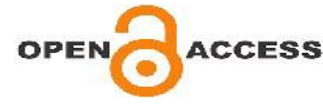


possible. Timely treatment with appropriate antibiotics before the urine culture and antibiogram results are very important in improving the patient and preventing kidney scarring and other complications [10]. In recent years, the overuse of common antibiotics has increased antibiotic resistance among urinary tract pathogens worldwide [10]. As a result, the most significant threat to public health is the issue of antibiotic-resistant bacteria, which can cause a high percentage of hospital deaths each year [11]. In Iran, the incidence of bacterial strains resistant to antibiotics in UTI is increasing and this issue is worrying [12]. It has been argued that local resistance to antibiotics should be assessed every five years for policy-making for the treatment of pediatric UTI [13]. This study aims to investigate the prevalence and profile of antibiotic resistance of bacterial agents isolated from children UTI of children admitted to Abuzar hospital in southwestern Iran.

\section{Materials and Methods}

This cross-sectional descriptive study was conducted from May 2020 to March 2021 on 1316 children admitted with UTI to Abuzar children's hospital in southwestern Iran. This study only included cases who had not received antibiotic treatment before hospitalization. Samples were collected in a sterile container and sent to the laboratory. The samples were cultured using a standard loop (0.01 ml) under sterile conditions on blood agar and Eosin methylene blue (EMB) agar (Himedia, India). After 18-24 hours of incubation at $37^{\circ} \mathrm{C}$, the culture media were evaluated for growth or lack of growth. Cultures with Candida growth were excluded. Gram-negative bacteria were identified by standard biochemical tests. Gram-positive microorganisms were identified with the corresponding laboratory tests: catalase, coagulase, and esculin agar (for enterococci).

The pattern of antimicrobial resistance of the studied strains was determined using the disc diffusion method by Mueller-Hinton medium (Himedia, India). According to the edge of the zone of growth inhibition encircling the discs, and based on Clinical and Laboratory Standards Institute (CLSI) recommendations [14], the results were classified as sensitive (S), intermediate-resistant (I) and, resistant (R). The antibiotics amikacin (AN, $30 \mu \mathrm{g}$ ), ampicillin (AM, $10 \mu \mathrm{g}$ ), ciprofloxacin (CP, $5 \mu \mathrm{g}$ ), gentamycin (GM, $10 \mu \mathrm{g}$ ), nitrofurantoin (FM, $300 \mu \mathrm{g}$ ), ceftriaxone (CRO,30 $\mu \mathrm{g}$ ), cefepime (FEP, $30 \mu \mathrm{g}$ ), erythromycin (E, $15 \mu \mathrm{g}$ ), imipenem (IMP,10 $\mu \mathrm{g}$ ), Cotrimoxazole (SXT, $1.25 / 23.75 \mu \mathrm{g}$ ), penicillin $(\mathrm{P}, 1 \mathrm{O} \mu \mathrm{g}$ ), cefoxitin (FOX, 30 $\mu \mathrm{g}$ ), piperacillin-tazobactam (TZP, 100/10 $\mu \mathrm{g}$ ), and CC (Clindamycin, $2 \mu \mathrm{g}$ ) (MAST Group Ltd., United Kingdom) were evaluated. For quality control of antibiogram steps in each series of experiments with clinical isolates, the sensitivity test was performed in the same way on the standard strains of $E$. coli ATCC 25922, Pseudomonas aeruginosa ATCC 27853, Staphylococcus aureus ATCC 25923, Enterococcus faecalis ATCC 29212 and the results were compared with standard values.

The results are shown as descriptive statistics in terms of relative frequency. The data were analyzed using the Chi-square test in SPSS software version 15. Statistical significance was considered as a $\mathrm{P}$ value $<0.05$.
Figure 1. Distribution of isolated organisms in children with UTI

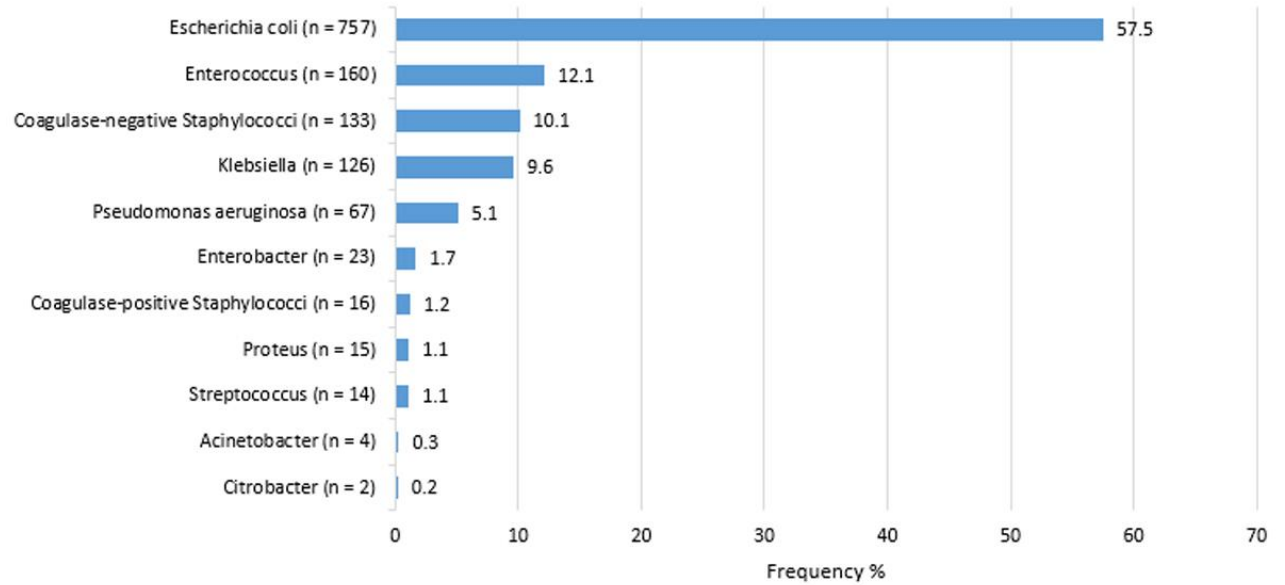




\section{Results}

Out of 1316 children with positive urine culture, 821 (62.39\%) were girls and 495 (37.61\%) were boys. The rate of UTI was higher in girls than boys and the difference was significant $(\mathrm{P}<0.05)$. The age of studied cases was up to 5 years old. The lowest incidence of UTI was observed in the age group under one year $(\mathrm{n}=57,4.33 \%)$.

The most prevalent strain isolated from urine cultures was $E$. coli (57.52\%) and the lowest strain was related to Citrobacter spp. (0.15\%) (Figure 1).
$E$. coli isolates showed the highest resistance (57.06\%) to ampicillin. Amikacin was recognized as the most effective antibiotic with $91.94 \%$ sensitivity against $E$. coli isolates. Tables 1 and 2 shows the distribution of antibiotic resistance of isolated bacteria.

\section{Discussion}

Urinary tract infections are the most common nosocomial infections, followed by surgical site infections, and lower respiratory tract infections $[15$, 16].

Table 1. Distribution of antibiotic resistance among Gram-negative bacteria in children with UTI *

\begin{tabular}{|c|c|c|c|c|c|c|c|c|}
\hline \multicolumn{2}{|c|}{ Antibiotics } & 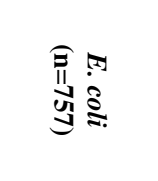 & 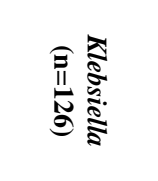 & 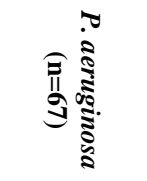 & 芯 & 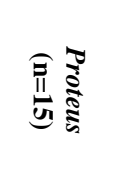 & 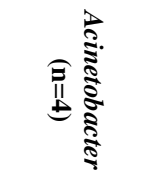 & 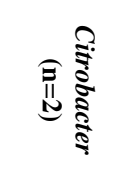 \\
\hline \multirow{3}{*}{ FM } & $\mathrm{S}$ & $559(73.8 \%)$ & $78(61.9 \%)$ & - & $9(39.1 \%)$ & - & - & - \\
\hline & I & $1(0.1 \%)$ & $2(1.5 \%)$ & - & $1(4.3 \%)$ & - & - & - \\
\hline & $\mathrm{R}$ & $22(2.9 \%)$ & $8(6.3 \%)$ & - & $5(21.7 \%)$ & - & - & - \\
\hline \multirow{3}{*}{ AN } & $\mathrm{S}$ & $696(91.9 \%)$ & $90(71.4 \%)$ & $59(88 \%)$ & $15(65.2 \%)$ & $2(50 \%)$ & $15(100 \%)$ & $2(100 \%)$ \\
\hline & I & $5(0.6 \%)$ & $4(3.1 \%)$ & $2(2.9 \%)$ & - & - & - & - \\
\hline & $\mathrm{R}$ & $22(2.9 \%)$ & $23(18.2 \%)$ & $1(1.4 \%)$ & $9(39.1 \%)$ & $2(50 \%)$ & - & $2(100 \%)$ \\
\hline \multirow{3}{*}{ CRO } & $\mathrm{S}$ & $343(45.3 \%)$ & $69(54.7 \%)$ & - & $12(52.1 \%)$ & - & $14(93.3 \%)$ & $2(100 \%)$ \\
\hline & I & $101(13.3 \%)$ & $3(2.3 \%)$ & - & - & - & - & - \\
\hline & $\mathrm{R}$ & $302(39.8 \%)$ & $46(36.5 \%)$ & - & $12(52.1 \%)$ & - & $1(6.6 \%)$ & $1(50 \%)$ \\
\hline \multirow{3}{*}{ GM } & $\mathrm{S}$ & $577(76.2 \%)$ & $91(72.2 \%)$ & $56(83.5 \%)$ & $14(60.8 \%)$ & $1(25 \%)$ & $15(100 \%)$ & $1(50 \%)$ \\
\hline & I & $4(0.5 \%)$ & - & - & - & - & - & - \\
\hline & $\mathrm{R}$ & $113(14.9 \%)$ & $23(18.2 \%)$ & $5(7.4 \%)$ & $8(34.7 \%)$ & $2(50 \%)$ & - & - \\
\hline \multirow{3}{*}{$\mathrm{AM}$} & $\mathrm{S}$ & $72(9.5 \%)$ & - & - & $2(8.6 \%)$ & - & - & - \\
\hline & I & - & - & - & - & - & - & - \\
\hline & $\mathrm{R}$ & $432(57 \%)$ & - & - & $15(65.2 \%)$ & - & - & - \\
\hline \multirow{3}{*}{ IMP } & $\mathrm{S}$ & $678(89.5 \%)$ & $106(84.1 \%)$ & $58(86.5 \%)$ & $23(100 \%)$ & $3(75 \%)$ & $13(86.6 \%)$ & $2(100 \%)$ \\
\hline & I & - & - & - & - & - & - & - \\
\hline & $\mathrm{R}$ & $3(0.3 \%)$ & $2(1.5 \%)$ & $2(2.9 \%)$ & - & $1(25 \%)$ & - & - \\
\hline \multirow{3}{*}{$\mathrm{CP}$} & $\mathrm{S}$ & - & $17(13.4 \%)$ & $28(41.7 \%)$ & $6(26 \%)$ & $1(25 \%)$ & $3(20 \%)$ & - \\
\hline & I & - & - & - & - & - & - & - \\
\hline & $\mathrm{R}$ & - & $2(1.5 \%)$ & $2(2.9 \%)$ & - & - & - & - \\
\hline \multirow{3}{*}{ FEP } & $\mathrm{S}$ & - & - & $5(7.4 \%)$ & - & - & - & - \\
\hline & I & - & - & - & - & - & - & - \\
\hline & $\mathrm{R}$ & - & $1(0.7 \%)$ & $2(2.9 \%)$ & - & - & - & - \\
\hline \multirow{3}{*}{$\mathrm{TZP}$} & $\mathrm{S}$ & - & - & $16(23.8 \%)$ & - & - & - & - \\
\hline & I & - & - & - & - & - & - & - \\
\hline & $\mathrm{R}$ & - & - & $2(2.9 \%)$ & - & $1(25 \%)$ & - & - \\
\hline
\end{tabular}

*Results estimated based on the number of tested/total isolates AN; Amikacin, AM; Ampicillin, CP; Ciprofloxacin, GM; Gentamycin, FM; Nitrofurantoin, CRO; Ceftriaxone, FEP; Cefepime, IMP; Imipenem, TZP; Piperacillin-tazobactam 
Table 2. Distribution of antibiotic resistance among Gram-positive bacteria in children with UTI ${ }^{*}$

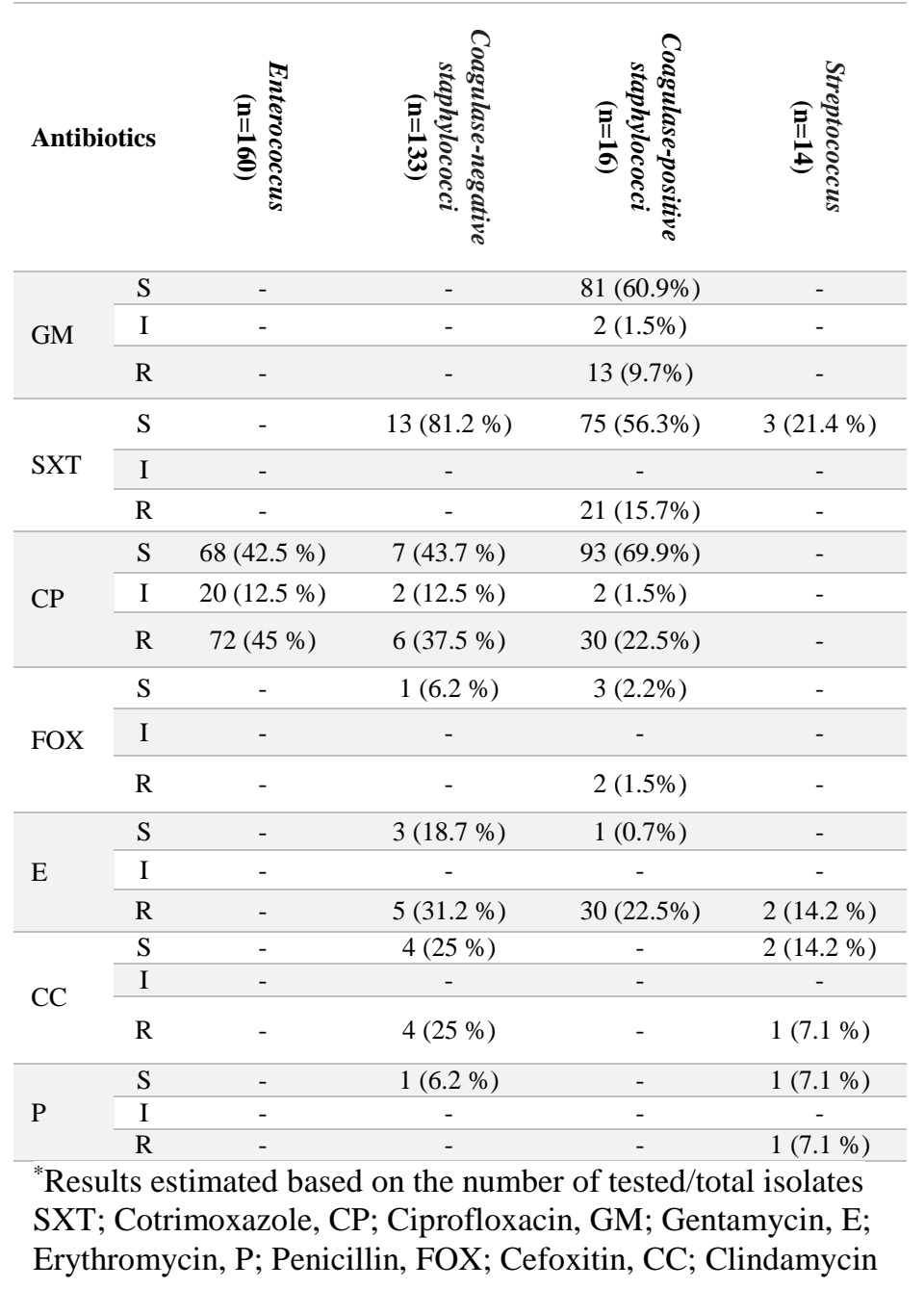

Proper treatment for this type of infection first requires careful examination of the drug resistance of the infectious agents and then drug administration. According to research, the sex ratio varies with age and during the first trimester, UTI is more prevalent in boys than in girls; while, at other ages, the prevalence of theinfection is higher in girls [17]. Here, the girls had the highest prevalence of UTI.

In the present study, consistent with other research, the most isolated strain from urine cultures was E. coli (57.52\%) [18-22]. However, by comparing the findings of this study with similar studies, we found differences in antibiotic resistance patterns in different regions.

Isolates of E. coli, Proteus and, Enterobacter were mostly resistant to ampicillin. Coagulase-negative staphylococci (CoNS) isolates were mostly resistant to erythromycin. It can be concluded that sensitivity to antibiotics has decreased due to irrational administration and use of drugs, enzymatic mutations, the transmission of resistance through genetic factors, and so on. Therefore, further studies in this field, decreasing the consumption of antibiotics, and promoting rational administration by physicians are essential.

Various microorganisms, such as E. coli, bind together to make a colonial tool that helps biofilms formation. Biofilms of microorganisms resist antimicrobial therapies. In a study by Valavi et al. in Ahvaz, Iran, E. coli was the most frequent pathogen isolated from children with UTI that showed the lowest resistance to nitrofurantoin [23]. In addition, Mehri et al. concluded that $E$. coli was the most isolated strain followed by Enterococcus; and nitrofurantoin and ceftriaxone were the antibiotics effective against bacterial agents [24]. These findings 
are consistent with our work. Barzan et al. in Tehran reported that Proteus isolates, like the isolates of the our study, showed full susceptibility to gentamicin [25].

Because, in different regions and over time, the frequency distribution of antibiotic resistance varies, it is recommended to carry out periodic monitoring of antibiotic resistance to control infection. This study is the only study on the antibiotic profile of the causes of UTIs in children in Ahvaz over the past 10 years. Examination of uropathogenic agents in each region helps physicians to update their knowledge about the causative agents of UTI tract infections, their susceptibility to antibiotics, and selecting an appropriate experimental treatment.

In summary, E. coli was the most common causative agent of UTI in children. Amikacin was recognized as the appropriate choice against urinary tract pathogens. Because, in different regions and over time, the frequency distribution of antibiotic resistance varies, it is recommended to carry out periodic monitoring of antibiotic resistance for infection control. Also, a widespread screening program for UTI in children should be introduced in order to determine the exact prevalence and rate of resistance of uropathogens to widely used antibiotics.

\section{Author contributions}

Conception or design of the work AY, SA; Data collection: SMM; Data analysis and interpretation AAZ, ZF; Drafting the article: MV; Critical revision of the article: AY. All authors read and approved the final version of manuscript.

\section{Conflict of interests}

The authors declare that they have no conflicts of interest.

\section{Ethical declarations}

The study was approved by the Student Research Committee, Ahvaz Jundishapur University of Medical Sciences, Ahvaz, Iran (No. IR.AJUMS.REC.1399.297).

\section{Financial Support}

This work was supported by a grant number 99530 from Vice Chancellor for Research, Ahvaz Jundishapur University of Medical Sciences.

\section{References}

1. Nugent J, Childers M, Singh-Miller N, Howard R, Allard R, Eberly M. Risk of Meningitis in Infants Aged 29 to 90 Days with Urinary Tract Infection: A Systematic Review and Meta-Analysis. J Pediatr. 2019; 212:102-10.e5.

2. Flores-Mireles AL, Walker JN, Caparon M, Hultgren SJ. Urinary tract infections: epidemiology, mechanisms of infection and treatment options. Nat Rev Microbiol. 2015; 13(5):269-84.

3. Bitsori M, Galanakis E. Pediatric urinary tract infections: diagnosis and treatment. Expert Rev Anti Infect Ther. 2012; 10(10):1153-64.

4. Mahmoudi H, Emadmomtaz H, Karimitabar Z, Emam AH, Alikhani MY. Prevalence of asymptomatic urinary tract infection in primary school children of Hamadan City and drug resistance of isolated microorganisms in 2014. Pajouhan Sci J. 2015; 13(3):8-14. 5. Kapur S, Bourke T, Maney JA, Moriarty P. Emergency department attendance following 4-component meningococcal B vaccination in infants. Arch Dis Child. 2017; 102(10):899-902.

6. Wagenlehner FME, Cloutier DJ, Komirenko AS, Cebrik DS, Krause KM, Keepers TR, et al. Once-Daily Plazomicin for Complicated Urinary Tract Infections. N Engl J Med. 2019; 380(8):729-40.

7. Dospinescu VM, Tiele A, Covington JA. Sniffing Out Urinary Tract Infection-Diagnosis Based on Volatile Organic Compounds and Smell Profile. Biosensors (Basel). 2020; 10(8).

8. Shrestha LB, Baral R, Poudel P, Khanal B. Clinical, etiological and antimicrobial susceptibility profile of pediatric urinary tract infections in a tertiary care hospital of Nepal. BMC Pediatr. 2019; 19(1):36.

9. Terlizzi ME, Gribaudo G, Maffei ME. UroPathogenic Escherichia coli (UPEC) Infections: Virulence Factors, Bladder Responses, Antibiotic, and Non-antibiotic Antimicrobial Strategies. Front Microbiol. 2017; 8:1566.

10. Kapur S, Gehani M, Kammili N, Bhardwaj P, Nag V, Devara SM, et al. Clinical Validation of Innovative Optical-Sensor-Based, Low-Cost, Rapid Diagnostic Test to Reduce Antimicrobial Resistance. J Clin Med. 2019; 8(12):2098.

11. Cassini A, Högberg LD, Plachouras D, Quattrocchi A, Hoxha A, Simonsen GS, et al. Attributable deaths and disability-adjusted lifeyears caused by infections with antibiotic-resistant bacteria in the EU and the European Economic Area in 2015: a population-level modelling analysis. Lancet Infect Dis. 2019; 19(1):56-66.

12. Zomorrodi A, Zargar M, Noroozi J. Evaluation of antibiotic resistance associated with ophthalmic oqxAB pumps in Klebsiella pneumoniae causing urinary tract infection. Med Sci J. 2019; 29(2):163-70.

13. Pape L, Gunzer F, Ziesing S, Pape A, Offner G, Ehrich JH. [Bacterial pathogens, resistance patterns and treatment options in community acquired pediatric urinary tract infection]. Klin Padiatr. 2004; 216(2):83-6.

14. CLSI (Ed.). Performance Standards for Antimicrobial Susceptibility Testing; 30th ed. CLSI Supplement M100. Clinical and Laboratory Standards Institute, Wayne, PA. 2020.

15. Saleem Z, Hassali MA, Godman B, Hashmi FK, Saleem F. A multicenter point prevalence survey of healthcare-associated infections in Pakistan: Findings and implications. Am J Infect Control. 2019; 47(4):421-4.

16. Izadi N, Eshrati B, Etemad K, Mehrabi Y, Hashemi-Nazari SS. Rate of the incidence of hospital-acquired infections in Iran based 


\section{Akrami et al.}

on the data of the national nosocomial infections surveillance. New Microbes New Infect. 2020; 38:100768.

17. Demir M, Kazanasmaz H. Uropathogens and antibiotic resistance in the community and hospital-induced urinary tract infected children. J Glob Antimicrob Resist. 2020; 20:68-73.

18. Düzgün A, Okumuş F, Saral A, Çiçek A, Cinemre S. Determination of antibiotic resistance genes and virulence factors in Escherichia coli isolated from Turkish patients with urinary tract infection. Rev Soc Bras Med Trop. 2019; 52:e20180499.

19. Jabrodini A, Heidari F, Taghavi SF, Shokouh MR. The Investigation of Frequency and Antibiotic Resistance Pattern of Escherichia coli and Klebsiella pneumoniae Isolated From Urinary Tract Infection in Outpatients Referred to Amiralmomenin Ali Hospital in Gerash City in 2017: A Short Report. J Rafsanjan Univ Med Sci. 2018; 17(1):75-84.

20. Norouzian H, Katouli M, Shahrokhi N, Sabeti S, Pooya M, Bouzari S. The relationship between phylogenetic groups and antibiotic susceptibility patterns of Escherichia coli strains isolated from feces and urine of patients with acute or recurrent urinary tract infection. Iran J Microbiol. 2019; 11(6):478-87.
21. Jena J, Sahoo RK, Debata NK, Subudhi E. Prevalence of TEM, SHV, and CTX-M genes of extended-spectrum $\beta$-lactamaseproducing Escherichia coli strains isolated from urinary tract infections in adults. 3 Biotech. 2017; 7(4):244.

22. Vranic SM, Zatric N, Rebic V, Aljicevic M, Abdulzaimovic A. The Most Frequent Isolates from Outpatients with Urinary Tract Infection. Mater Sociomed. 2017; 29(1):17-20.

23. Valavi E, Nikfar R, Ahmadzadeh A, Kompani F, Najafi R, Hoseini R. The Last Three Years Antibiotic Susceptibility Patterns of Uropathogens in Southwest of Iran. Jundishapur J Microbiol. 2013; 6(4):e4958.

24. Mehri T, Tahereh Z-K, Khadijeh M, Parisa A, Majid A. Urinary Tract Infection and its Antibiotic Sensitivity in Acute Childhood Diarrhoea. Int J Med Res Health Sci. 2017; 6(8):65-8.

25. Barzan M, Hoseyni-Doust R, Ghalavand Z. Investigation of frequency and antimicrobial pattern of gram-negative bacteria isolated from urine specimens of children with urinary tract infection in Tehran, Iran. Iran J Med Microbiol. 2016; 9(4):99-104. 